The Israeli Journal of Aquaculture IJA.73.2021.1427476, 12 pages

CCBY-NC-ND-4.0 • https://doi.org/10.46989/001c.25698

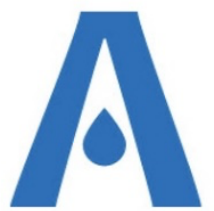

The $I J A$ is a peer-reviewed open-access, electronic journal, freely available without charge to users

Produced by the AquacultureHub non-profit Foundation Sale of $I J A$ papers is strictly forbidden

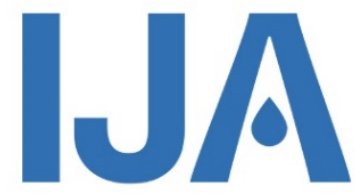

\title{
Analysis of gut microbiota and immune-related genes during sea cucumber (Apostichopus japonicus) response to dietary supplementation with Codonopsis pilosula
}

\section{Xiaolu Wang ${ }^{1}$, Ying Fan ${ }^{1}{ }^{*}$ Xiaoqing $\mathbf{Y u}^{1}$, Youhong Wang ${ }^{1}$, Haibin $\mathrm{Ye}^{1}$, Jing Diao ${ }^{1}$, Shuxian Wang ${ }^{1}$, Hongjun Liu ${ }^{1}$, La $\mathrm{Xu}^{1}{ }^{1}$, Chunlei Gai ${ }^{1}$, Danping $\mathrm{Ma}^{1}$, Tao Zhang ${ }^{2}$} ${ }^{1}$ Marine Science Research Institute of Shandong Province, Shandong Key Laboratory of
Disease Control in Mariculture, Qingdao 266100, China

${ }^{2}$ Wudi Municipal Marine Development Bureau

Key words: Codonopsis pilosula, Apostichopus japonicas, gut microbiota, highthroughput sequencing, immunity

\begin{abstract}
The gut microbiota composition of sea cucumber (Apostichopus japonicas) was investigated using high-throughput sequencing techniques. The mRNA expression of complement component 3 and lysozyme genes was evaluated using quantitative fluorescence PCR. Sea cucumbers were fed with a basal diet (control group) and an experimental diet supplemented with Codonopsis pilosula (experimental group) for 30 days. The results showed that the alpha diversity of the gut microbiota was changed in different indices, including Chao1, the abundance-based coverage estimator, the Shannon index, and Good's coverage. Dietary C. pilosula promoted the proliferation of the Flavobacteriaceae family of the Proteobacteria phylum and reduced the relative abundance of the Verrucomicrobiaceae family of the Verrucomicrobia phylum. We concluded that dietary $C$. pilosula supplementation could alter the network interactions among different microbial functional groups by changing the ecological network's microbial community composition and biological evolution. A positive effect on $A$. japonicus immune responses in the gut was seen via increasing the mRNA expression of the complement component 3 and lysozyme genes. It seems to happen via modulating the balance in gut microbiota.
\end{abstract}

* Corresponding author. e-mail: fy_fy123@126.com 


\section{Introduction}

The animal gut is a complex and powerful immune organ. It harbors complex communities of microbes, which play a critical role in the host's health by improving digestion or protecting against pathogenic bacterial infections (Clemente et al., 2012). There are trillions of bacteria in animals' guts, interacting with each other and forming complicated networks that accomplish systems functions through the flow of energy, matter, and information. In recent years, because of their high abundance and nutritional value, bacteria in the gut have been considered as either a direct source of disease or a source that indirectly provides the host with essential nutrients (Amaro et al., 2009; Gao et al., 2010). With the rapid development of high throughput sequencing technologies, genetic information has provided deep insights into microbiota. The association between diet and health is vital and accepted by aquaculture researchers. For example, a study using fatty acid biomarkers showed that bacteria were important dietary components of the sea cucumber, Apostichopus japonicus (Gao et al., 2010). Gut microbiota in the holothurians, A. japonicus and Holothuria leucospilota were shown to produce extracellular enzymes that could degrade indigestible products such as polysaccharides (Zhang et al., 2012; Zhang et al., 2013). Other reports suggested that gut bacteria may play a role in supplying specific essential amino acids to holothurians (Phillips, 1984). Researchers well accept the strong association between diet, microbes, and health. However, no reports have studied the interactions and changes among gut microbiota after $A$. japonicus is fed $C$. pilosula.

The sea cucumber is an economically important aquaculture species in China. Recently $A$. japonicus has become one of the most expensive and highly-demanded sea foods, and the farming of $A$. japonicas in northern China is essential for fisheries (Jia et al., 2020; Song et al., 2019). The rapid expansion and industrialization of sea cucumber farming have been negatively affected by infectious diseases, which have caused substantial economic losses (Wang et al., 2007). However, potential preventive and therapeutic strategies for sea cucumber infections are unavailable due to limited research efforts. Existing dietary supplementation strategies, especially the use of immunostimulants, may be applied to the control and prevention of $A$. japonicas disease. The composition and diversity of microorganisms in the digestive tract of $A$. japonicus have been surveyed sporadically. For example, Zhang et al. (2011) investigated the microorganism composition in the digestive tract of $A$. japonicus using traditional culturedependent methods. Gao et al. (2010) analyzed bacterial diversity in the digestive tract of $A$. japonicus cultured in a pond, and found differences between the anterior intestine, middle intestine, and posterior intestine. Song et al. (2019) suggested that dietary Astragalus polysaccharide (APS) supplementation changed the enzymatic activity and expression levels of immune-related genes and antioxidant-related genes. They significantly changed the relative abundances of Proteobacteria and Bacteroidetes in the intestines of sea cucumber.

Therefore, this study aimed to evaluate the effects of dietary C. pilosula supplementation for the gut microbiota of sea cucumber by using high-throughput sequencing techniques. We identified a role for $C$. pilosula as a new feed additive. Lysozyme (LSZ) is an efficient natural antimicrobial enzyme and is one of the most critical immune factors in aquatic animals. LSZ can effectively damage the cell walls of pathogens invading hosts and plays an essential role in the innate immune defense (Wang et al., 2011). The complement system has been discovered in invertebrates and vertebrates, and plays a crucial role in the host's innate defense against common pathogens (Zhou et al., 2011). As a central component in the complement system, complement component 3 (C3) is an intermediary between the innate and adaptive immune systems. Thus, the mRNA expression of $\mathrm{C} 3$ and lys was evaluated further to summarize $C$. pilosula on the immunity of sea cucumber. 


\section{Materials and Methods}

Experimental animals and culture conditions

Disease-free healthy sea cucumbers were obtained from a sea cucumber farm (Qingdao, China). Sea cucumbers were cultured in a $60 \mathrm{~L}$ fiberglass tank and fed a basal diet (Great seven Bio-Tech, Qingdao, China) for $7 \mathrm{~d}$ to acclimate to the experimental conditions. Following a $24 \mathrm{~h}$ fast, sea cucumbers of similar size $(19.0 \pm 2.0 \mathrm{~g}$, means \pm $\mathrm{SE})$ were randomly distributed into five aquaria at a density of 12 sea cucumbers per aquarium. Sea cucumbers were fed once daily at 3:00 pm for 30 days. The feeding rates were $2 \%$ of the bodyweight during the feeding trial. The temperature was $16 \pm 2{ }^{\circ} \mathrm{C}$, the $\mathrm{pH}$ was $8.0 \pm 0.5$, the salinity was $30 \%$, and the dissolved oxygen was $>5 \mathrm{mg} / \mathrm{L}$. Onehalf of the water volume in the recirculating system was replaced by fresh seawater once per day, and all water was replaced once per week to maintain the water quality.

Experimental diets and design

C. pilosula was provided by Qingdao Guofeng Pharmaceutical Co., Ltd. (Qingdao, China) and crushed to 300 mesh before use. The marine mud and Sargassum thunbergii were provided by Qingdao Saigelin Bioengineering Co., Ltd (Qingdao, China). The basal diet was a 1:1 mixture of marine mud and Sargassum thunbergia. The experimental diet was made by adding $C$. pilosula at $2 \%$ of the mass of Sargassum thunbergia to the basal diet. Sea cucumbers in the control group (CT) were fed with the basal diet, while sea cucumbers in the experimental group (EP) were fed the experimental diet. CT and EP had three replicates.

Sample collection and processing

On the $28^{\text {th }}$ day of the experiment, three sea cucumbers from each aquarium were removed at random. Their guts were isolated and immediately placed on an ice plate in a sterile environment. The gut contents were then removed through sterile operation. The contents were then collected and placed into a sterile Eppendorf centrifuge tube, and immediately placed in liquid nitrogen until experimentation. A portion of the contents were used for mRNA expression analyses, while another was used for gut microbiota analysis.

Gut microbiota analysis

According to the manufacturer's instructions, Bacterial DNA was extracted using a TIANamp Stool DNA Kit (Tiangen Biotech, Beijing, China). Amplification and sequencing of the $V 3$ region of the bacterial 16S DNA gene were performed using primers $\mathrm{F} 357\left(5^{\prime}-\right.$ CCTACGGGAGGCAGCAG-3') and R518 (5'ATTACCGCGGCTG CTGG-3'). PCR amplification was performed under the following conditions: initial denaturation at $94^{\circ} \mathrm{C}$ for $2 \mathrm{~min}, 30$ cycles of $94^{\circ} \mathrm{C}$ for $30 \mathrm{~s}, 52^{\circ} \mathrm{C}$ for $30 \mathrm{~s}$ and $72^{\circ} \mathrm{C}$ for $90 \mathrm{~s}$, with an extension time increase of $1 \mathrm{~s}$ over each cycle. A final extension of $72^{\circ} \mathrm{C}$ for 5 min was also performed. All samples were amplified in triplicate, and all products were purified using the AxyPrep PCR Cleanup Kit (Axygen, USA). The purified PCR products were quantified using a Quant-iT PicoGreen dsDNA Assay Kit on the Promega QuantiFluor Fluorescence Quantitative System. The library concentration was more than $2 \mathrm{nM}$. After gradient dilution, the qualified libraries were mixed in proportion according to the required quantities and sequenced by $\mathrm{NaOH}$ denaturation as a single chain. The final library was sequenced using the Illumina Miseq 2 $\times 300$ bp paired-end platform. From the original data, the joint sequences were removed; the paired-end reads were merged into a long tag, and tags with $\mathrm{N}$ (undetermined base information) $\geq 5 \%$ and inferior quality (quality $Q<10$ and base $>20 \%$ of the whole tag) were removed to obtain clean data. Operational taxonomic units (OTUs) were defined with a threshold of $97 \%$ similarity. Then, a representative OTU sequence was assigned to a taxonomic identity using an RDP classifier with a default confidence threshold of 0.8-1.0. Of the remaining OTUs, only those for which at least five reads were recovered across all samples were retained for further analyses.

The relative abundance of bacteria was calculated using the sum of all tags corresponding to one bacterial group from the replicates, divided by the sum of all tags of all bacterial groups from the replicates. Alpha diversity between bacterial communities was evaluated by Shannon, ACE, Chao1, and Goods coverage. The mean of the estimated richness was used for comparisons between different samples. 
mRNA expression analysis

According to the manufacturer's instructions, total RNA was isolated using the UNIQ-10 Column Total RNA Isolation Kit (Sangon, Shanghai, China). The quality and quantity of the extracted RNA were measured using the Ultramicro Nucleic Acid Analyzer (Biodropsis BD-1000) and agarose gel electrophoresis. First-strand cDNA synthesis was performed in a volume of $20 \mu \mathrm{L}$ with $900 \mathrm{ng}$ of total RNA, $25 \mathrm{pM}$ of oligo dT primers, 50 $\mathrm{pM}$ of random hexamers, $1 \times$ PrimeScript buffer, and $0.5 \mathrm{~mL}$ of PrimeScript RT enzyme Mix I (PrimeScript RT reagent Kit, TaKaRa, China). Reactions were incubated at $37^{\circ} \mathrm{C}$ for $15 \mathrm{~min}$, and then at $85^{\circ} \mathrm{C}$ for $5 \mathrm{~s}$ to deactivate the enzyme.

Equal amounts of CDNA templates were used for quantitative real-time PCR ( qRT-PCR was performed in $20 \mu \mathrm{L}$ reactions containing the following components: $10 \mu \mathrm{L}$ of $2 \times$ SYBR Green Master mix (SYBR PrimeScript RT-PCR Kit II, TaKaRa), $0.4 \mu \mathrm{L}$ of ROX Reference Dye II, $1 \mu \mathrm{L}$ of cDNA template, and $0.4 \mathrm{mM}$ of each primer (Table 1). The qRT-PCRs were performed in triplicate using the CFX96 Real-Time System (Bio-RAD). The qRT-PCR parameters were as follows: one cycle of $95^{\circ} \mathrm{C}$ or $30 \mathrm{~s}$, followed by 40 cycles of $95^{\circ} \mathrm{C}$ for $10 \mathrm{~s}, 56^{\circ} \mathrm{C}$ for $25 \mathrm{~s}$ and $72^{\circ} \mathrm{C}$ for $25 \mathrm{~s}$. Melting curve analyses of the amplification products were performed at the end of each PCR reaction to confirm that only one PCR product was amplified and detected. In addition, the amplicon size was confirmed by agarose gel electrophoresis with a 100 bp ladder. The cytochrome b (Cytb) gene was used as the reference. The relative mRNA levels of the target genes were calculated as $2^{-\Delta \Delta \mathrm{Ct}}$ (Livak and Schmittgen, 2001).

Statistical analysis

The gut microbiota data were analyzed using mean values. The RT-qPCR data were expressed as the mean \pm standard error $(S E)$. Duncan's multiple range test tested the differences in SPSS 17.0. The level of significance was set at $P<0.05$. All of the data were tested for normality, homogeneity, and independence before ANOVA.

Table 1 PCR primers used in this study

\begin{tabular}{lll}
\hline Prime & Sequence (5' to $3^{\prime}$ ) & Sequence information \\
\hline Aj-C3-F & GCGTTGTTCGTTCAACAAGGGGA & For AjC3 Real-time PCR \\
Aj-C3-R & GCCATTCACTGGAGGTGTGGCA & \\
Aj-LSZ-F & AGGGAGGTAGTCTGGATGGA & For Aj-LSZ Real-time PCR \\
Aj-LSZ-R & GCGCAAAATCCTCACAGGTA & \\
Cytb-F & TGAGCCGCAACAGTAATC & For Real-time PCR \\
Cytb-R & AAGGGAAAAGGAAGTGAAAG & \\
\hline
\end{tabular}

\section{Diversity of gut microbiota}

\section{Results}

The number of OTUs, the Chao1, abundance-based coverage estimator (ACE), and Shannon indices were obtained for all samples to assess the alpha diversity of gut microbiota in sea cucumbers in the two groups (Table 2). All indices suggested that there were different diversities between the CT group and the ET group. C. pilosula optimized the gut microbiota. C. pilosula supplementation may inhibit the growth of some bacteria. We also computed the Good's coverage. On average, the two groups had a Good's coverage of 0.971 and 0.966 , respectively, indicating that roughly two additional OTUs would be expected for every 100 additionally-sequenced reads.

Composition of gut microbiota.

QIIME was used to randomly sample the total number of sequences in the operable taxon abundance matrix at different depths. The sparse curve (Figure 1) was drawn according to the number of sequences extracted at each depth and the corresponding operable taxon number. With the further increase of the number of sequences randomly selected, the curve of each sample tended to be flat. The results showed that the effective sequencing quantity had been able to cover the diversity of bacteria, the sampling depth 
was reliable, and the sequencing results could truly reflect the quantity relationship of dominant bacteria in the sample.

Table 2 Diversity indices used in this study (means $\pm \mathrm{SE}$ )

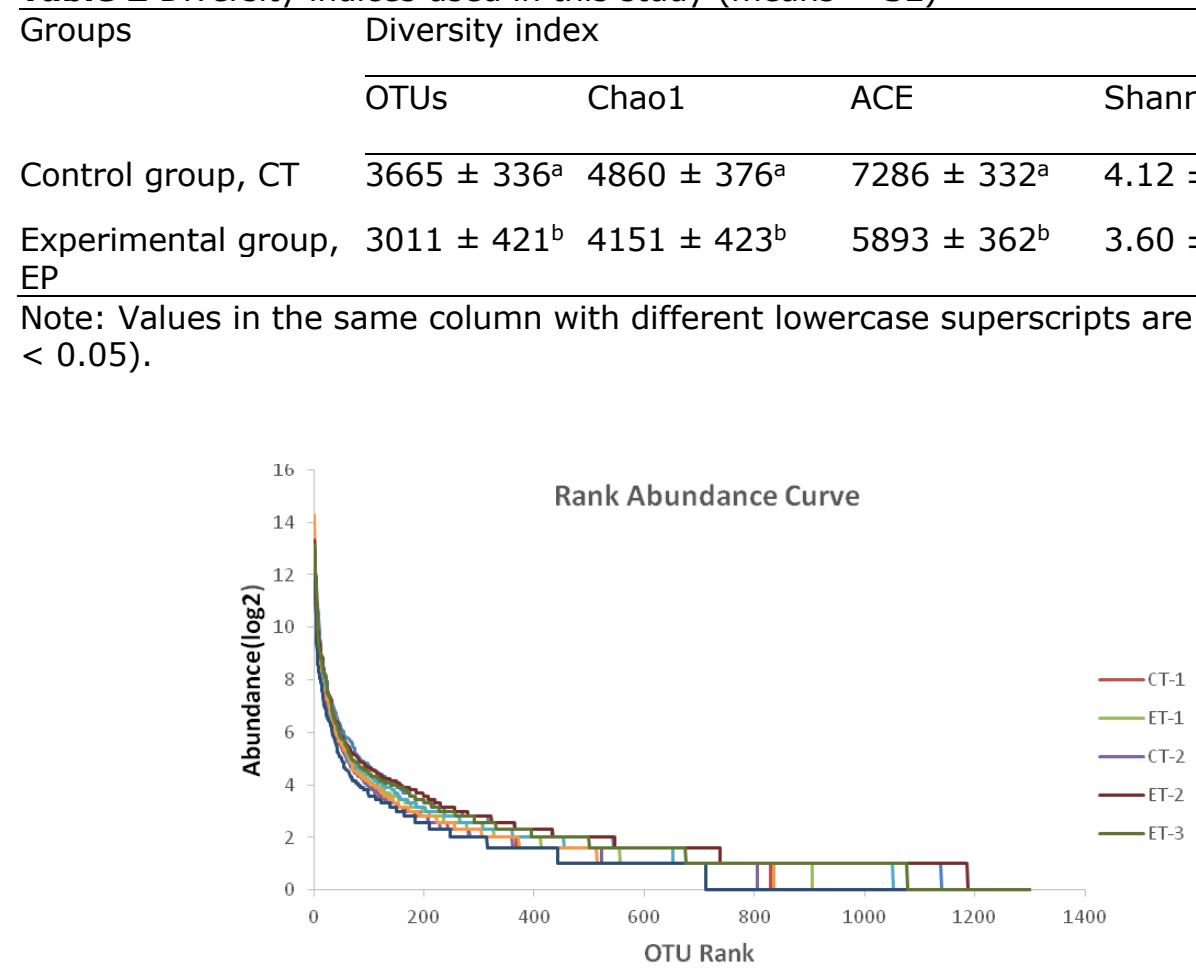

Figure 1 Diversity comparison

Taxonomically, 34 different bacterial phyla or groups in the sea cucumber gut were identified (Table 3) (Figure 2). The dominant phyla in both groups were Proteobacteria, Bacteroidetes, and Verrucomicrobia. Specifically, as shown in Table 3, the CT group contained Bacteroidetes (8.8\%), Proteobacteria (25.1\%), and Verrucomicrobia $(57.4 \%)$, while the EP group contained Bacteroidetes (21.4\%), Proteobacteria $(28.1 \%)$, and Verrucomicrobia (39.1\%).

Table 3 The abundance of different bacterial phyla in the gut of $A$. japonicus [(\%) means]

\begin{tabular}{lll}
\hline Bacteria & Control group (CT) & Experimental group (EP) \\
\hline Verrucomicrobia & 57.4 & 39.1 \\
Sphingobacteria & 0.5 & 1.4 \\
Proteobacteria & 25.1 & 28.1 \\
Chlorobi & 3.4 & 4.7 \\
Firmiaites & 1.2 & 1.2 \\
Cyanobacteria & 0.8 & 0.8 \\
Bacteroidetes & 8.8 & 21.4 \\
Actinobacteria & 1.8 & 1.2 \\
Acidobacteria & 0.8 & 1.1
\end{tabular}

The relative abundance of Proteobacteria and Bacteroidetes increased by dietary supplementation of $C$. pilosula, which was concurrent with the reduction in Verrucomicrobia. 


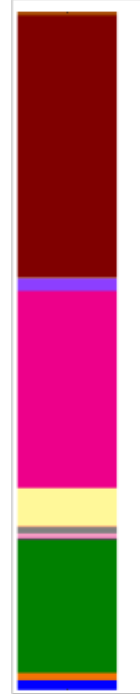

ET-1

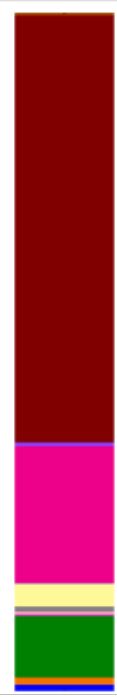

CT-1

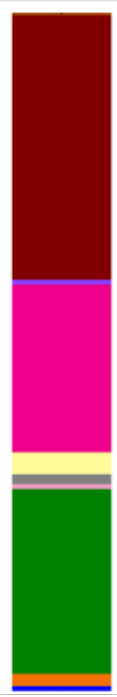

ET-2

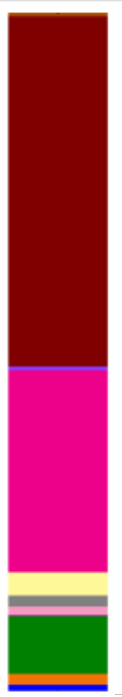

CT-2

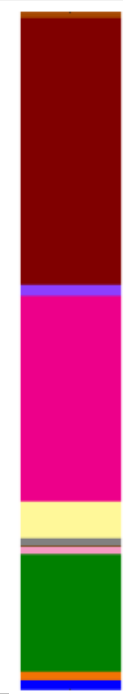

ET-3

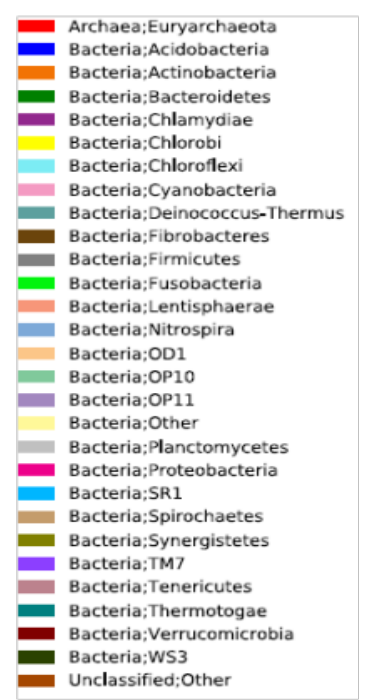

Unclassified;Othe

Figure 2 Microbial communities in phylum level

The abundance results of genus level were consistent with that of phylum level (Figure 3). The higher abundance was streptophyta in the Verrucomicrobia (36.8\%), the second was Rhodobacteraceae in Proteobacteria (13.9\%).

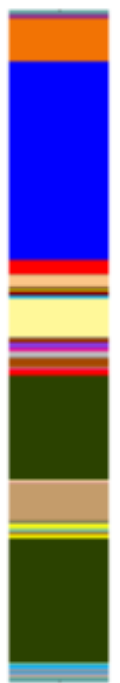

ET-1

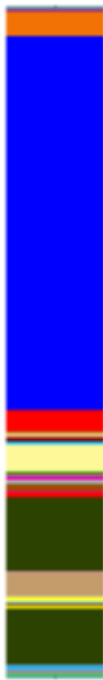

CT-1

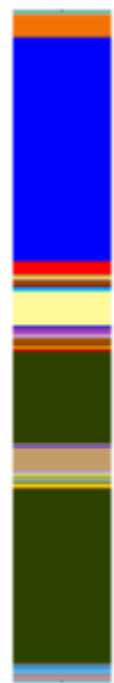

ET-2

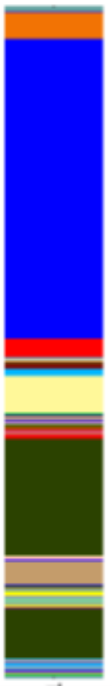

CT-2

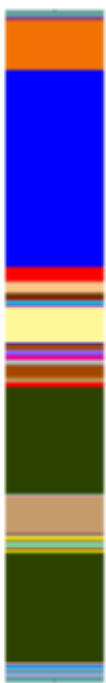

ET-3

Bacteria;Verrucomicrobia;Verrucomicrobiae;Verrucomicrobiales;Rubritaleaceae;Rubritalea

Bacteria;Bacteroidetes;Flavobacteria;Flavobacteriales;Flavobacteriaceae;Other

- Bacteria;Proteobacteria;Alphaproteobacteria;Rhodobacterales;Rhodobacteraceae;Other Bacteria;Proteobacteria;Gammaproteobacteria;Other;Other;Other

- Bacteria;Verrucomicrobia;Verrucomicrobiae;Verrucomicrobiales;Other;Other

Bacteria;Verrucomicrobia;Verrucomicrobiae;Verrucomicrobiales;Verrucomicrobiaceae;Haloferula

Bacteria;Cyanobacteria;Cyanobacteria;Chloroplast;Streptophyta;Other

Bacteria;Proteobacteria;Deltaproteobacteria;Desulfobacterales;Desulfobacteraceae;Other

Figure 3 Microbial communities in genus level 
According to the principal coordinate PCoA analysis, the change situation and change rate of species among communities were shown in Figure 4, and the range of diversity coefficient was within $14.91 \% \sim 15.47 \%, 15.47 \% \sim 16.21 \%, 14.91 \% \sim 16.21 \%$.
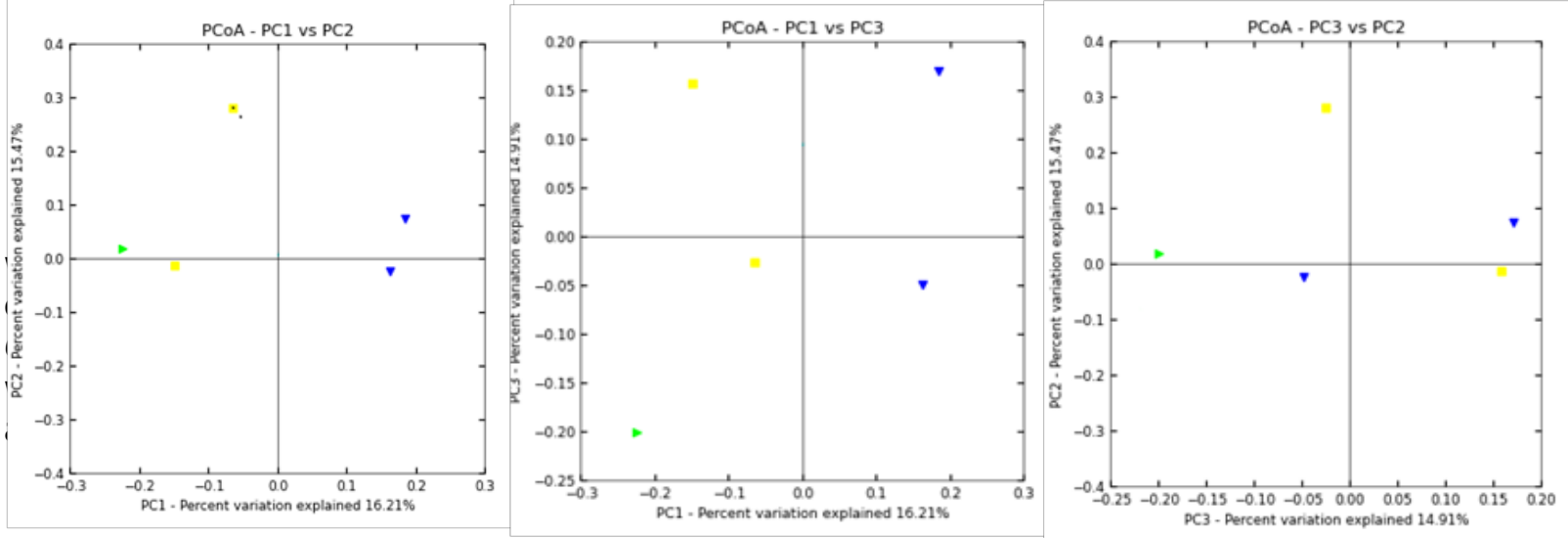

Figure 4 2D PCoA graph in PCoA analysis (percentage of diversity)

Figure 5 presents the analysis of the relationship among the bacterial diversity of each sample expressed in a VENN diagram. There were 733 standard operable units between control and experimental groups in the gut samples after administering the Chinese herbal medicine C. pilosula. In comparison, there were 1198 different operable units in the control group, and there were 870 other operable units. In brief, dietary $C$. pilosula supplementation dramatically altered the interaction relationship.

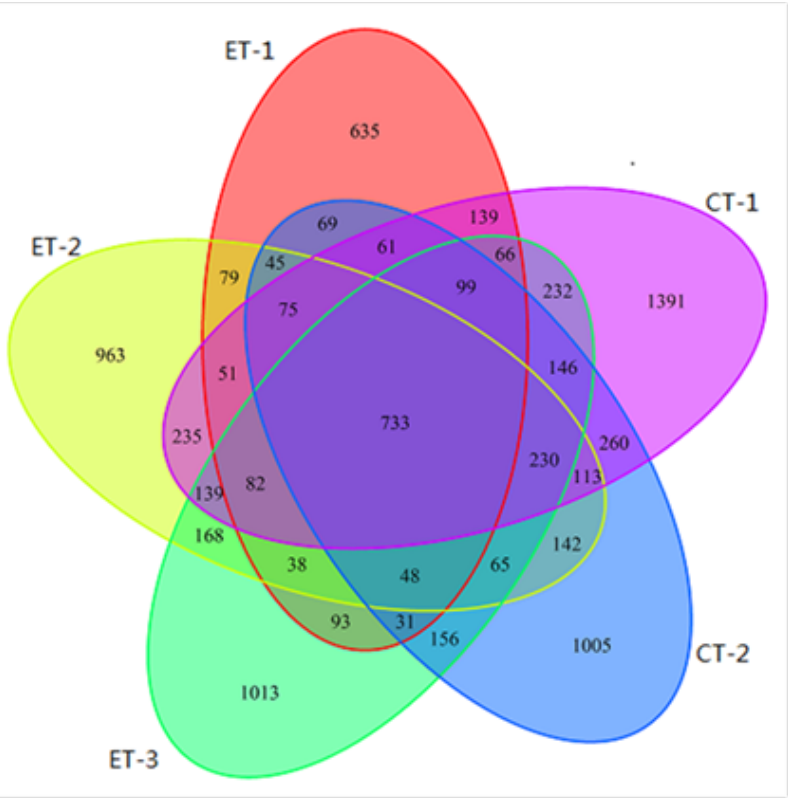

Figure 5 VENN diagram of the OTU Intersections 
There was a positive impact on the immunity of sea cucumber. After dietary $C$. pilosula supplementation, the relative expression levels of the Aj-C3 and Aj-LSZ genes in the gut tissue of sea cucumber in the EP group were significantly higher than those in the CT group on the $28^{\text {th }}$ day $(P<0.05)$ (Table 4$)$.

Table 4 The relative expression of immune genes in the gut of $A$. japonicus (means $\pm \mathrm{SE}$ )

\begin{tabular}{lll}
\hline Groups & $A j-C 3$ & $A j-l y s$ \\
\hline Control group, CT & $1.44 \pm 0.57^{\mathrm{a}}$ & $1.16 \pm 0.12^{\mathrm{a}}$ \\
Experimental group, EP & $2.43 \pm 0.06^{\mathrm{b}}$ & $1.89 \pm 0.09^{\mathrm{b}}$ \\
\hline
\end{tabular}

Note: Values in the same column with different lowercase superscripts are significantly different $(P<0.05)$.

\section{Discussion}

Chinese herbal medicines are well known as immune enhancers. In aquaculture, Chinese herbal medicines can inhibit pathogens, improve growth and immunity, promote digestion, and improve the structure of the intestinal microbiota (Chang et al., 2017; Chen et al., 2018; Wang et al., 2017). C. pilosula is a type of Chinese herb that contains active ingredients, such as polysaccharides, phenols, sterols, and saponins, among others. Such ingredients would play a positive role in the immune and digestion of sea cucumber. It has been reported that polysaccharides, which are major components of $C$. pilosula have many bioactivities, including immuno-enhancement, antitumor, antioxidant, and hypolipidemic (Sun and Liu, 2008). Thus, C. pilosula can replace antibiotics, inhibit pathogenic microorganisms, regulate the gut environment, and is safer than antibiotics (Zhao et al., 2013). However, a few polysaccharides have been studied in sea cucumber (Fan et al., 2013; Zhang et al., 2011; Zhao et al., 2011) and the results of those studies should be considered during the administration of polysaccharides.

In the present study, $C$. pilosula influenced interactions between species. From the microbiome viewpoint, OTUs of the same species within a module likely shared the same functions, consistent with the findings of Yan et al. (2014). Different indexes (e.g., Chao1, ACE, Shannon, Good's coverage) represented different meanings, and showed different effects. The Chao1 and ACE indexes indicate different community richness. The Shannon index indicates different community diversities. Good's coverage calculates of the number of OTUs with abundance of 1 and estimates the influence of low abundance. These indexes showed that the contents of $C$. pilosula promoted the proliferation of dominant bacteria and inhibited the growth of few bacteria; thus, adjusting the balance of the microbiota and indirectly regulating the immunity and health of sea cucumbers.

In addition to the changes in gut microbial diversity, the composition of gut microbes changed as well. The dominant phyla in both groups were Proteobacteria, Bacteroidetes, and Verrucomicrobia. At the class level, the dominant bacterial in the CT group were Verrucomicrobiae, Proteobacteria, and Bacteroidetes, whereas the dominant bacteria in the EP group were Alphaproteobacteria, Flavobacteriia, and Verrucomicrobiae. The reasons for such differences might be due to $C$. pilosula optimizing the gut environment by adding nutrients or altering micro-metabolism. A considerable role for the gut microflora is the processing and assimilation of food. It was similar to the findings of Bogatyrenko and Buzoleva (2016), Gao et al. (2014) and Yang et al. (2015). Those studies found that different dominant groups likely resulted from differences in diets. Compared to the control group, C. pilosula supplementation changed the nutritional composition in the gut, which led to changes in the flora, but did not increase the abundance of Verrucomicrobia. Little is known about the metabolism and ecological roles of Verrucomicrobia, except that they can degrade polysaccharides (Cardman et al., 2014). Proteobacteria and Bacteroidetes increased significantly following C. pilosula supplementation. The difference between these phyla and Verrucomicrobiaceae was likely because few to no Bifidobacterium spp. or Lactobacillus spp. are found in the sea cucumber, and $C$. pilosula was used as their nutrient source, which accelerated the growth of the bacteria. 
A heat map was generated after classifying the sequencing data at the genus level and it was found that $C$. pilosula changed the composition of the microbiota. The similarity between the CT and EP groups was still high. Beta diversity based on the community structure was used to compare the differences among samples, and reflected the environmental heterogeneity. The specific mechanism must be further studied.

During the coevolution of intestinal microbial communities and hosts, some genes involved in host microbial interactions will be increased. Such genes have particular specificity to host intestinal microorganisms, which can be referred to in the functional network. A network connection between OTUs described the co-occurrence among microbiomes, which might be due to species performing similar or complementary functions (Zhou et al., 2011). Bacteria in the gut interact with each other, form complicated networks, and perform functions through the flow of energy, matter, and information. Thus, it is important to understand the functional network structures and the underlying mechanisms, an essential part of ecology (Olesen et al., 2007).

In addition to the change in microbial community composition, the module hubs in the EP group were also different from those in the CT group. This was probably due to the negative impact of $C$. pilosula supplementation on intestinal bacterial diversity in the EP group. From the molecular viewpoint, OTUs of the same species within a module likely share the same functions (Yan et al., 2014). Negative interactions may indicate competition or predation among the taxa, while positive interactions signify complementation or cooperative behaviors. The analysis of modular topological roles was important to identify key microbial groups based on the OTUs' roles in their modules (Deng et al., 2012). Structurally, the networks would not be affected by the extinction of peripherals.

Conversely, connectors and module hubs could play an indispensable role in the network. In brief, those results suggested that the dietary supplementation of $C$. pilosula regulated the balance of intestinal microbiota in sea cucumbers via changes in microbial community composition. However, the details of the functions of the microbiota in the ecological network require mechanistic studies. Apart from that, the strains of the typical sea cucumber microbiota characterized by the ecological network may be good probiotics for use in aquaculture (Bogatyrenko et al., 2010).

Intestine immunity plays a vital role in protecting sea cucumber from different pathogens and environmental stresses. Sea cucumbers lack an adaptive immune system and their defense mechanisms mainly rely on innate immune responses to protect them against diseases. The diverse microbial communities in the gut play a critical role in the host's health, including influencing immunity, nutrient processing, and homeostasis (Becattini et al., 2016). It was reported that the intake of exogenous substances could damage the intestinal structure, increase the abundance of pathogenic bacteria, and stimulate the responses of the inflammatory and immune systems. Therefore, it is crucial to improve intestinal immunity to resist pathogen challenge (Duan et al., 2017; Suo et al., 2017). LSZ plays a crucial role in mediating protection against bacterial invasion in the presence of complement (Duan et al., 2016). Complement components also play a pivotal role in immune responses toward bacterial infections (Gao et al., 2010). Although the heterogeneous nature of gut contents might result in different gene expression patterns, the primary purpose of this study was to gain a broad understanding of gut health in response to $C$. pilosula. We performed analyses of gene expression and provided insights into critical immune pathways and processes. In the present study, dietary supplementation with $C$. pilosula significantly induced and improved the mRNA expression of $\mathrm{Aj}-\mathrm{LSZ}$ and $\mathrm{Aj}-\mathrm{C} 3$ in the gut of sea cucumber. Those data suggested that C. pilosula might influence the intestinal immune responses of sea cucumber to protect intestinal health. The bioactivity of Codonoposis polysaccharide could participate in metabolic processes and promote the expression of immune-related genes. Similar results were found in a previous study where Astragalus membranaceus and its polysaccharides beneficially affected the immune system of sea cucumber (Wang et al., 2009). However, how dietary $C$. pilosula supplementation modulates the innate immune responses via alterations in the sea cucumber gut microbiota requires further investigation. 
In conclusion, our study demonstrated that dietary supplementation of C. pilosula could modulate the gut microbial community in sea cucumber by promoting the proliferation of Proteobacteria and Bacteroidetes. It also changed the topological roles of the OTUs, and increased the mRNA expression of complement component 3 and lysozyme genes in the gut.

\section{Acknowledgements}

We would like to thank the National Surveillance program on Aquatic Animal Diseases, and the Surveillance plan of aquatic animal diseases in Shandong Province. We also thank Dr. Chen for reviewing the manuscript. We thank International Science Editing (http://www.internationalscienceediting.com) for editing the manuscript.

\section{References}

Amaro, T., Witte, H., Herndl, G. J., Cunha, M. R., Billett, D. S., 2009. Deep-sea bacterial communities in sediments and guts of deposit-feeding holothurians in Portuguese canyons (NE Atlantic). Deep Sea Research, I 56: 1834-1843. https://doi.org/10.1016/j.dsr.2009.05.014

Becattini, S., Taur, Y., Pamer, E.G., 2016. Antibiotic-induced changes in the intestinal microbiota and disease. Trends in Molecular Medicine, 22 (6): 458-478. https://doi.org/10.1016/j.molmed.2016.04.003

Bogatyrenko, E. A., Buzoleva, L. S., Chi, Z., 2010. Potential probiotics of the Far Eastern trepang Apostichopus japonicas producing digestive enzymes. Microbiology (Moscow), 79(2): 173-177. https://doi.org/10.1134/S0026261710020074

Bogatyrenko, E. A., Buzoleva, L. S., 2016. Characterization of the gut bacterial community of the Japanese sea cucumber Apostichopus japonicas. Microbiology, 85 (1), 116-123. https://doi.org/10.1134/S0026261716010033

Cardman, Z., Arnosti, C., Durbin, A., Ziervogel, K., Teske, A., 2014. Verrucomicrobia are candidates for polysaccharide-degrading bacterioplankton in an arctic fjord of Svalbard. Applied and Environmental Microbiology, 80: 37493756. https://doi.org/10.1128/AEM.00899-14

Chang, Z. Q., Ge, Q. Q., Sun, M., Wang, Q., Lv, H. Y., Li, J., 2017. Immune responses by dietary supplement with Astragalus polysaccharides in the Pacific white shrimp. Litopenaeus vannamei. Aquaculture Nutrition, 24(2), 702-711. https://doi.org/10.1111/anu.12599

Chen, J., Ren, Y., Li, Y., Xia, B., 2018. Regulation of growth, intestinal microbiota, nonspecific immune response and disease resistance of sea cucumber Apostichopus japonicus (Selenka) in biofloc systems. Fish \& Shellfish Immunology, 77(7): 175-186. https://doi.org/10.1016/j.fsi.2018.03.053

Clemente, J. C., Ursell, L. K., Parfrey, L.W., Knight, R., 2012. The impact of the gut microbiota on human health: an integrative view. Cell, 148: 1258-1270.

https://doi.org/10.1016/j.cell.2012.01.035

Deng, Y., Jiang, Y.H., Yang, Y., He, Z., Luo, F., Zhou, J., 2012. Molecular ecological network analyses. BMC Bioinformatics, 13: 113.

https://doi.org/10.1186/1471-2105-13-113

Duan, Y. F., Zhang, J.S., Dong, H. B., Wang, Y., Liu, Q. S., Li, H., 2016. Effect of desiccation and resubmersion on the oxidative stress response of the kuruma shrimp Marsupenaeus japonicas. Fish \& Shellfish Immunoogyl, 49: 91-99.

https://doi.org/10.1016/j.fsi.2015.12.018

Duan, Y.F., Zhang, Y., Dong, H.B., Wang, Y., Li, H., Liu, Q. S., 2017. Intestine oxidative stress and immune response to sulfide stress in Pacific white shrimp Litopenaeus vannamei. Fish \& Shellfish Immunology, 63: 201-207.

https://doi.org/10.1016/j.fsi.2017.02.013

Fan, Y., Yu, X. Q., Xu, L., Wang, S. X., Ye, H. B., Diao, J., Yang, X. S., Li, T.B., 2013. Synergy of microcapsules polysaccharides and Bacillus subtilis on the growth, immunity and resistance of sea cucumber, Apostichopus japonicus against Vibrio splendidus infection. Fisheries Sciences, 79: 807-814. https://doi.org/10.1007/s12562-013-0644-3 
Fei, G., Li, F., Jie, T., Yan, J., Sun, H., Badger, J. H., 2014. Bacterial community composition in the gut content and Ambient Sediment of sea cucumber Apostichopus japonicas revealed by 16S rRNA gene Pyrosequencing. Plos One, 9: e100092.

https://doi.org/10.1111/jwas.12633

Gao, F., Xu, Q., Yang, H., 2010. Seasonal variations of food sources in Apostichopus japonicus indicated by fatty acid biomarkers analysis. Journal of Fishery Sciences of China, 34 760-767. [in Chinese] https://doi.org/10.3724/SP.J.1231.2010.06768

Gao, F., Sun, H.L., Xu, Q., Tan, J., Yan, J.P., Wang, Q. Y., 2010. PCR-DGGE analysis of bacterial community composition in the gut contents of Apostichopus japonicas. Journal of Fishery Sciences of China, 17: 672-680. https://doi.org/10.3724/SP.J.1011.2010.01351

Jia, C.C., Zhu, X.Z., Meng, X.Y., Yi, Y.T., 2020. Effects of Jerusalem artichoke powder on the growth performance, nonspecific immunity, and gastrointestinal microbiota of sea cucumber. Journal of the World Aquaculture Society, 51: 200-213.

https://doi.org/10.1111/jwas.12633

Livak, K. J., Schmittgen, T. D., 2002. Analysis of relative gene expression data using real-time quantitative PCR. Methods, 25: 402-408.

https://doi.org/10.1006/meth.2001.1262

Olesen, J, M., Bascompte, J., Dupont, Y. L., Jordano, P., 2007. The modularity of pollination networks. Proceedings of the National Academy of Sciences of the Unites States of America, 104: 19891-19896. https://doi.org/10.1073/pnas.0706375104

Phillips N. W.1984. Role of different microbes and substrates as potential suppliers of specific essential nutrients to marine detritivores. Bulletin of Marine Science, 35: 283-298. https://doi.org/10.1515/botm.1984.27.12.571

Song, X. J., Feng, Z. F., Zhang, Y. P., Zhu, W., 2019. Regulation of dietary astragalus polysaccharide (APS) supplementation on the non-specific immune response and intestinal microbiota of sea cucumber Apostichopus japonicas. Fish \& Shellfish Immunology, 94: 517524. https://doi.org/10.1016/j.fsi.2019.09.049

Sun, Y. X., Liu, J. C., 2008. Structural characterization of a water-soluble polysaccharide from the roots of Codonopsis pilosula and its immunity activity. International Journal of Biological Macromolecules, 43: 279-282.

https://doi.org/10.1016/j.ijbiomac.2008.06.009

Suo, Y. T., Li, E. C., Li, T. Y., Jia, Y. Y., Qin, J. G., Gu, Z. M., 2017. Response of gut health and microbiota to sulfide exposure in Pacific white shrimp Litopenaeus vannamei. Fish \& Shellfish Immunology, 63: 87-96. https://doi.org/10.1016/j.fsi.2017.02.008

Wang, P. H., Chang, Y. Q., Yu, J. H., Li, C. Y., Xu, G. R., 2007. Acute peristome edema disease in juvenile and adult sea cucumbers Apostichopus japonicus (Selenka) reared in North China. Journal of Invertebrate Pathology, 96: 11-17. https://doi.org/10.1016/j.jip.2007.03.001

Wang, T. T., Sun, Y. X., Jin, L. J., Xu, Y. P., Wang, L., Ren, T. J., Wang, K. L., 2009. Enhancement of non-specific immune response in sea cucumber (Apostichopus japonicus) by Astragalus membranaceus and its polysaccharides. Fish \& Shellfish Immunology, 27: 757-762. https://doi.org/10.1016/j.fsi.2009.09.002

Wang, T. T., Xu, Y. P., Liu, W. J., Sun, Y. X., Jin, L. J., 2011. Expression of Apostichopus japonicus lysozyme in the methylotrophic yeast Pichia pastoris. Protein Expression and Purification, 77: 20-25. https://doi.org/10.1016/j.pep.2011.01.002

Wang, X., Sun, Y., Wang, L., Li, X., Qu, K., Xu, Y., 2017. Synbiotic dietary supplement affects growth, immune responses and intestinal microbiota of Apostichopus japonicas. Fish \& Shellfish Immunology, 68(9): 232-242.

https://doi.org/10.1016/j.fsi.2017.07.027

Yan, K. K., Wang, D., Rozowsky, J., Zheng, H., Cheng, C., Gerstein, M., 2014. OrthoClust: an orthology-based network framework for clustering data across multiple species. Genome Biology, 15: R100.

https://doi.org/10.1186/gb-2014-15-8-r100 
Yang, G., Xu, Z. J., Tian, X. L., Dong, S.L., Peng, M.,2015. Intestinal microbiota and immune related genes in sea cucumber (Apostichopus japonicus) response to dietary $\beta$ glucan supplementation. Biochemical and Biophysical Research Communications, 458: 98103. https://doi.org/10.1016/j.bbrc.2015.01.074

Zhang, W. J., Hou, H. M., Zhang, G. L., Li, Q. Y., Du, C.M., 2011. Study on diversity of intestine cultivable microorganisms from Apostichopus japonicas. Science and Technology of Food Industry, 32: 149-155.

https://doi.org/10.1097/RLU.0b013e3181f49ac7

Zhang, Q., Mai, K. S., Zhang, W. B., Ma, H. M., Ai, Q. H., Xu, W., Liufu, Z. G., 2011. Effects of dietary selenoyeast and vitamin $\mathrm{E}$ on growth, immunity and disease resistance of sea cucumbers (Apostichopus japonicus Selenka). Chinese Journal of Animal Nutrition, 23: 1745-1755. https://doi.org/10.1016/S1671-2927(11)60313-1

Zhang, X., Nakahara, T., Miyazaki, M., Nogi, Y., Taniyama, S., Arakawa, O., 2012. Diversity and function of aerobic culturable bacteria in the intestine of the sea cucumber Holothuria leucospilota. The Journal of General and Applied Microbiology, 58: 447-456. https://doi.org/10.1080/09583157.2012.735223

Zhang, X., Nakahara, T., Murase, S., Nakata, H., Inoue, T., Kudo, T., 2013. Physiological characterization of aerobic culturable bacteria in the intestine of the sea cucumber Apostichopus japonicas. The Journal of General and Applied Microbiology, 59: 110. https://doi.org/10.2323/jgam.59.1

Zhao, Y. C., Ma, H. M., Zhang, W. B., Ai, Q. H., Mai, K. S., Xu, W., Wang, X. J., Liufu, Z. G., 2011. Effects of dietary $\beta$-glucan on the growth, immune responses and resistance of sea cucumber, Apostichopus japonicus against Vibrio splendidus infection. Aquaculture, 315: 269-274. https://doi.org/10.1016/j.aquaculture.2011.02.032

Zhao, X. N., Hu, Y. L., Wang, D. Y., Liu, J. Z., Guo, L. W., 2013. The comparison of immune-enhancing activity of sulfated polysaccharidses from Tremella and Condonpsis pilosula. Carbohydrate Polymers, 98: 438-443. https://doi.org/10.1016/j.carbpol.2013.06.043

Zhou, J., Deng, Y., Luo, F., He, Z., Yang, Y., 2011. Phylogenetic molecular ecological network of soil microbial communities in response to elevated $\mathrm{CO}_{2}$. MBio, 2: 122-111. https://doi.org/10.1128/mBio.00122-11

Zhou, Z. C., Sun, D. P., Yang, A. F., Dong, Y., Chen, Z., Wang, X. Y., Guan, X. Y., Jiang, B., Wang, B., 2011. Molecular characterization and expression analysis of a complement component 3 in the sea cucumber (Apostichopus japonicus). Fish \& Shellfish Immunology, 31: 540-547. https://doi.org/10.1016/j.fsi.2011.06.023 